\title{
Astrometry by small ground-based telescopes
}

\author{
W. Thuillot ${ }^{1}$, M. Stavinschi ${ }^{2}$, M. Assafin ${ }^{3}$ and the IAU Working \\ Group ASGBT † \\ ${ }^{1}$ Institut de mécanique céleste et de calcul des éphémérides, IMCCE-Paris Observatory \\ 77, av. Denfert Rochereau, 75014, Paris, France \\ email: thuillot@imcce.fr \\ ${ }^{2}$ Astronomical Institute of the Romanian Academy, AIRA, \\ Str. Cutitul de Argint, 5, Bucharest, Romania \\ email: magda@aira.astro.ro \\ ${ }^{3}$ Observatório Nacional, MCT, \\ R. Gal. José Cristino, 77 20921-400, So Cristóvo, Rio de Janeiro, Brazil \\ email: massaf@ov.ufrj.br
}

\begin{abstract}
Many small ground-based telescopes (with diameter less than $2 \mathrm{~m}$ ) allow us to perform programs of observations well adapted to astrometric measurements. The improvement of limiting magnitudes thanks to the use of CCD detector and their availability make them very useful for follow-up programs or observations on alert. This communication gives several examples of research carried out by members of the IAU working group "Astrometry by small ground-based telescopes". We also propose setting up of a network of observers for the Gaia follow-up observations.
\end{abstract}

Keywords. astrometry, surveys, telescopes, asteroids, occultations

\section{Introduction}

What is the interest to use small telescopes for astrometry nowadays when we can access new technologies on large telescopes or in space? What will be the use of small telescopes for astrometry once the Gaia mission provides a variety parameters for astrophysical objects and Solar System bodies with a never reached accuracy? Our Working Group intends to give the answers to these questions, by collecting information on research programs carried out and on scientific results obtained thanks to these instruments. The WG would also like to promote new astrometric programs.

\section{Overview}

Small telescopes (up to $2 \mathrm{~m}$ diameter) are numerous and generally easier to access than the larger ones. These characteristics are precious and we can get benefit from them by performing well adapted programs, in particular, observations on alert or long term programs. A census organized in 2006 by our working group showed that many programs are active. Some of them will be no more relevant after the Gaia mission but may

$\dagger$ Astrometry by Small Ground-Based Telescopes Working Group members: Andrei A., Arlot, J.-E., Pinigin G., Bazey N., Gontcharov G., Gumerov R., Jin Wenjing, Muinos Haro J., Niarchos P., Pereira Osorio J., Pascu D., Pauwels Th., Prostyuk Y., Pugliano A., Rafferty Th., Russell J. L., Rylkov V., Sanchez M., Shulga A., Souchay J., Tang Z. H., Teixeira R., Upgren A., van Altena W., Vieira Martins R., Zacharias N 
nevertheless contribute to its preparation. Our website at http://www/imcce.fr/astrom provides this list.

\section{Several international cooperations}

Several projects are based on the use of the small telescopes leading to international cooperation. We can for example mention the following examples. Astrometry for the prediction of stellar occultation can be a key program in order to organize the campaign for these events. The small telescopes can carry out the photometric observations and guarantee the success thanks to the longitude and latitude coverage. Such events have been successfully predicted and observed recently by Sicardy et al. (2006), in particular thanks to $60 \mathrm{~cm}$ to $1.6 \mathrm{~m}$ size telescopes of the Laboratorio Nacional de Astrofysica (LNA, Itajuba, Brazil) by the Rio Group (Assafin M., Andrei A., Vieira-Martins R., Veiga C. and colleagues from Observatorio Nacional/MCT and Observatorio do Valongo). Small telescopes can perform the photometry of mutual events of natural satellites which are currently predicted for the Galilean system, but also the Saturnian and the Uranian satellites. A campaign is organized by Arlot et al. (2006) and lightcurves will give highly accurate astrometric measurements. Mutual events of satellites of asteroids can also be performed to get the measurement of shape, size, and the determination of orbital elements (Descamps et al. 2007). Another international collaboration is involved in the CCD astrometry of ICRF radio sources. The Rio Group and astronomers from the Astronomical Institute of the Romanian Academy and USNO currently use small size telescopes in this work (Assafin et al. 2003).

\section{A ground-based network for a Gaia follow-up}

Small telescopes are involved in the organization of a ground-based network for the Gaia follow-up. This network will have the goal to supplement some Gaia observations for specific Solar System objects (Thuillot 2005). Supplementary astrometric observations could be necessary for some fast moving Near Earth Asteroids, Inner-Earth orbit asteroids, for improvement of orbits and photometric measurements could be necessary for objects suspected in cometary activity. Furthermore, astrometric observations will be useful to supplement the observations of asteroids by Gaia gravitationally deflected during asteroid/asteroid encounters on dates close to the edge of the mission (Mouret et al. 2007). Setting up such a ground-based network of observers dedicated to the Gaia follow-up is in progress. Several candidate sites are already identified but new locations are welcome in order to cover a large span of longitudes and latitudes.

\section{References}

Arlot, J.-E., Lainey, V., \& Thuillot, W. 2006, AA, 456, 1173

Assafin, M., Monken Gomes, P. T., da Silva Neto, D. N., Andrei, A. H., Vieira Martins, R., Camargo, J. I. B., Teixeira, R. \& Benevides-Soares, P. 2005, AJ, 129, 2907

Descamps, P., Vieira Martins, R. (2007), Icarus, 187, 482

Mouret, S., Hestroffer, D. \& Mignard, F. 2007, AA, 472, 1017.

Sicardy, B., Bellucci, A., Gendron, E., Lacombe, F., Lacour, S., Lecacheux, J., Lellouch, E., Renner, S., Pau, S., Roques, F. \& 35 coauthors 2006, Nature, 439, 52

Thuillot, W. 2005, in C. Turon, K. S. O'Flaherty \& M. A. C. Perryman (Eds.), Proc. Gaia Symposium The Three-Dimensional Universe with Gaia (ESA SP-576), p. 317 